

\title{
Genome Sequencing in Cancer Research and Therapy
}

\author{
Stefan Fröhling
}

National Center for Tumor Diseases (NCT) Heidelberg

German Cancer Research Center (DKFZ)

Heidelberg University Hospital

IMBS Symposium: Science, Ethics and Society

Freiburg, August 28, 2015

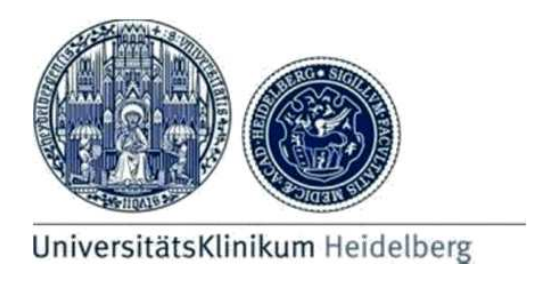




\section{Cancer Genome Sequencing Pathogenetic Insights and Clinical Impact}

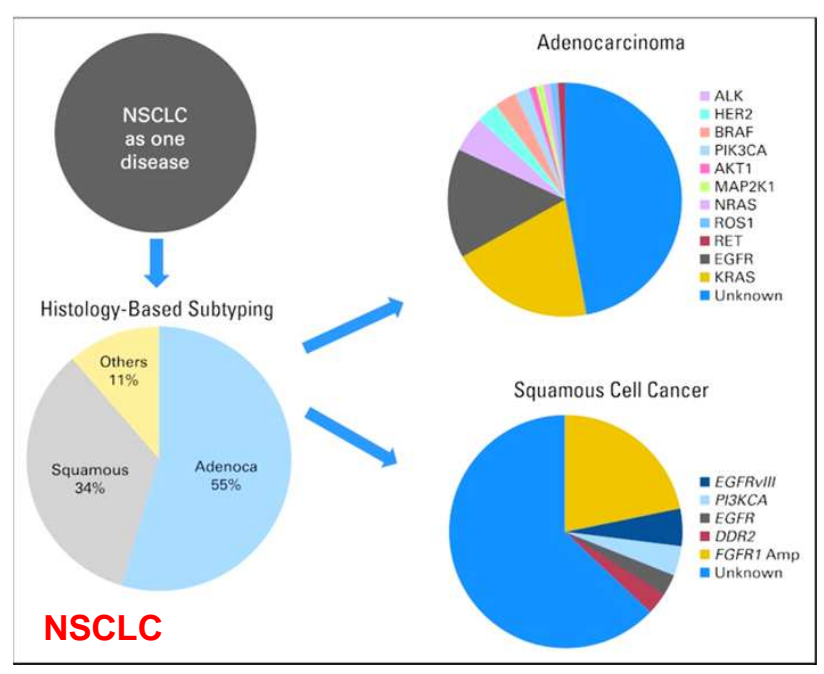

Common cancers as multiple rare diseases of the same organ, demanding unique therapies Li et al. J Clin Oncol 2013

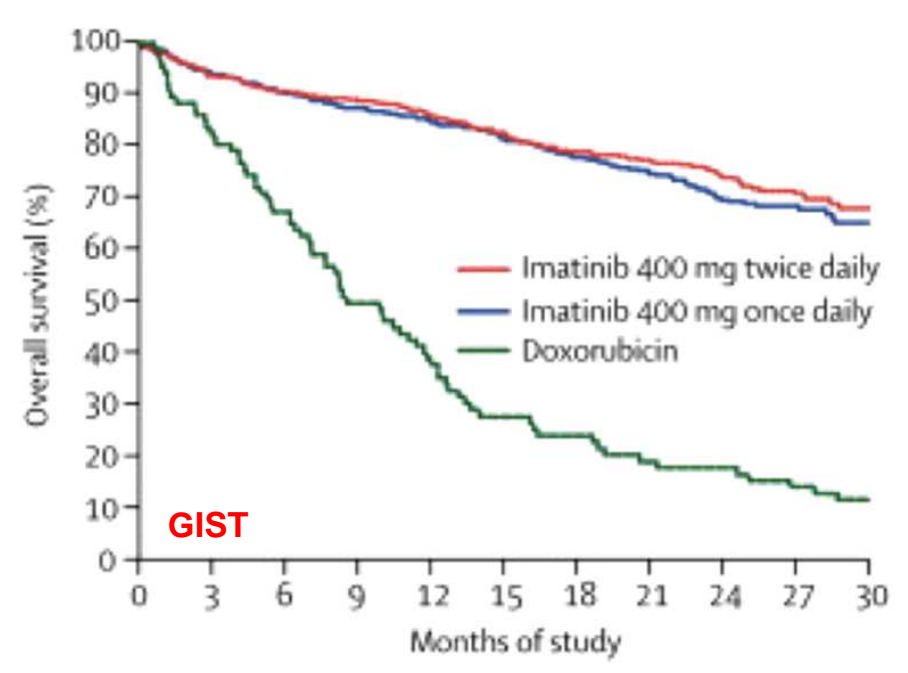

Improved clinical outcome through genotype-directed therapy Verwej et al. Lancet 2004

Distinct mutations shared across multiple cancers $B R A F^{\mathrm{V} 600 \mathrm{E} / \mathrm{K}}$ in melanoma; thyroid, lung, colorectal, ovarian, gastric, esophageal, head and neck cancer; gastrointestinal stromal tumor; glioma; hairy-cell leukemia; multiple myeloma; etc. 


\section{Cancer Genome Sequencing}

$$
\text { "N = 1" Studies }
$$

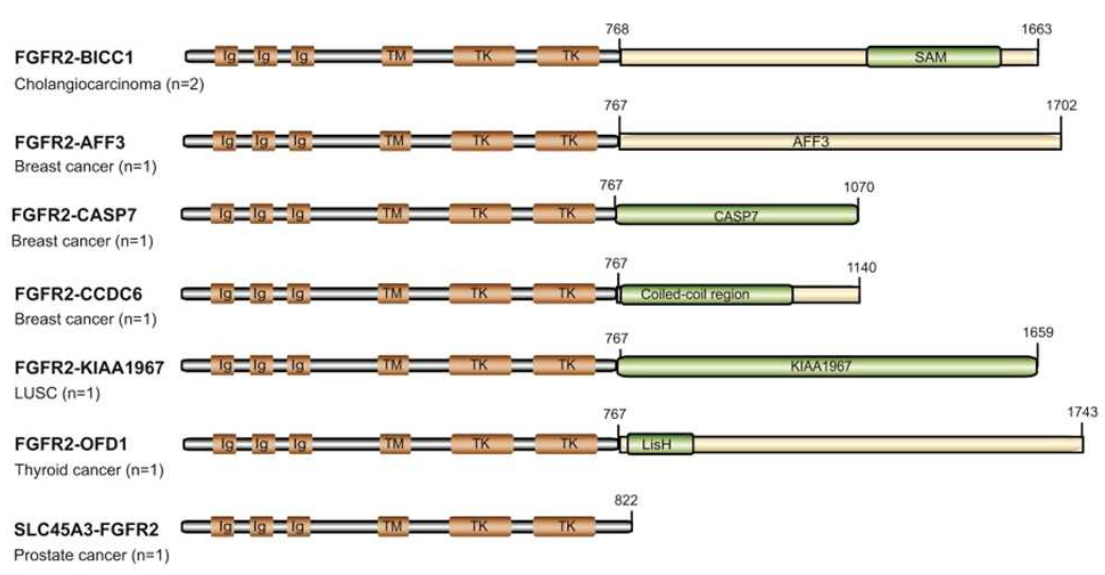

Targetable FGFR fusions in diverse cancers Wu et al. Cancer Discov 2013

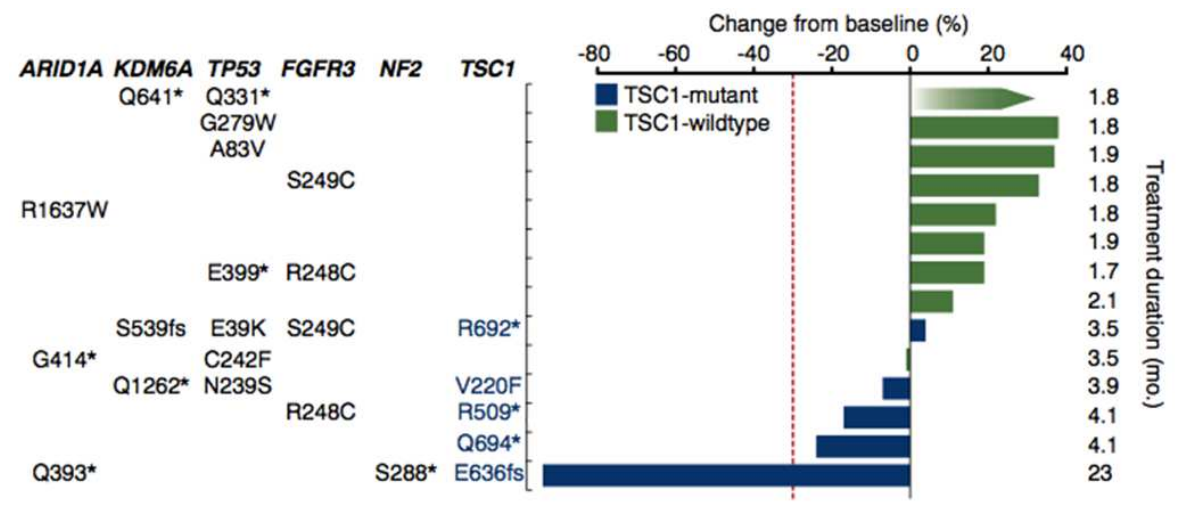

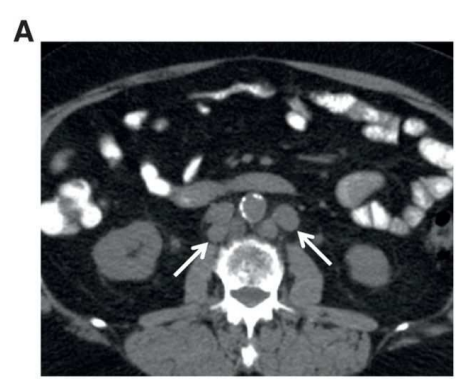
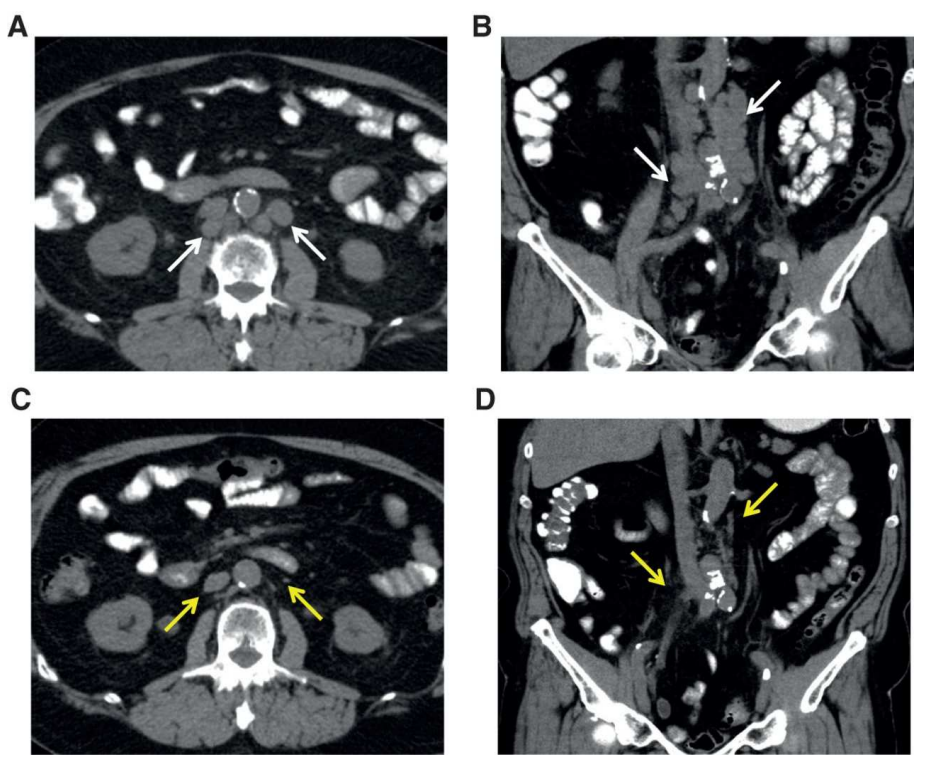

Activating mTOR mutations in urothelial carcinoma

Wagle et al. Cancer Discov 2014

Inactivating TSC1 mutations in urothelial carcinoma

lyer et al. Science 2012 


\section{Cancer Genome Sequencing Importance of Rare Mutations}

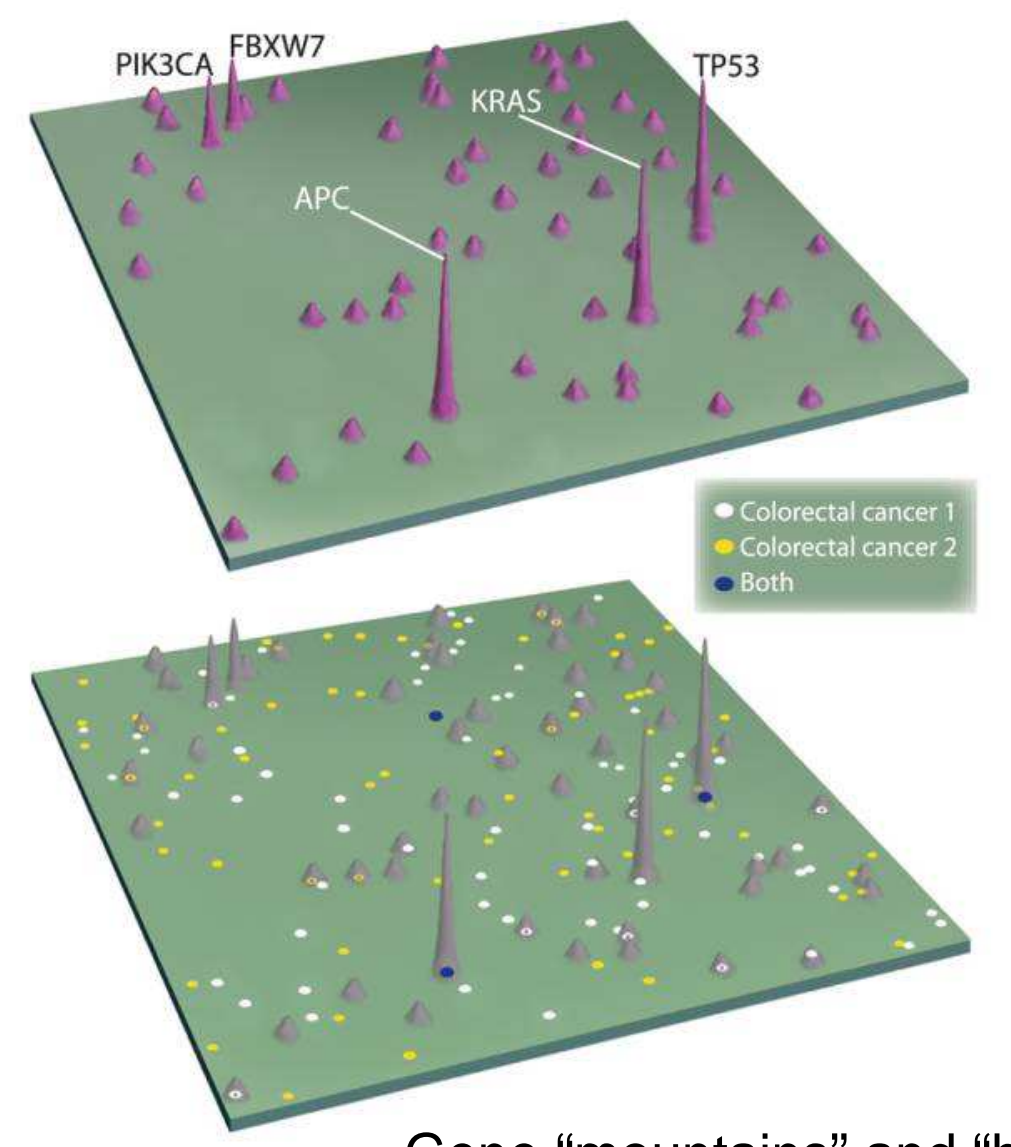

Gene "mountains" and "hills"

Wood et al. Science 2007
The Cancer Genome Atlas

Understanding genomics to improve cancer care

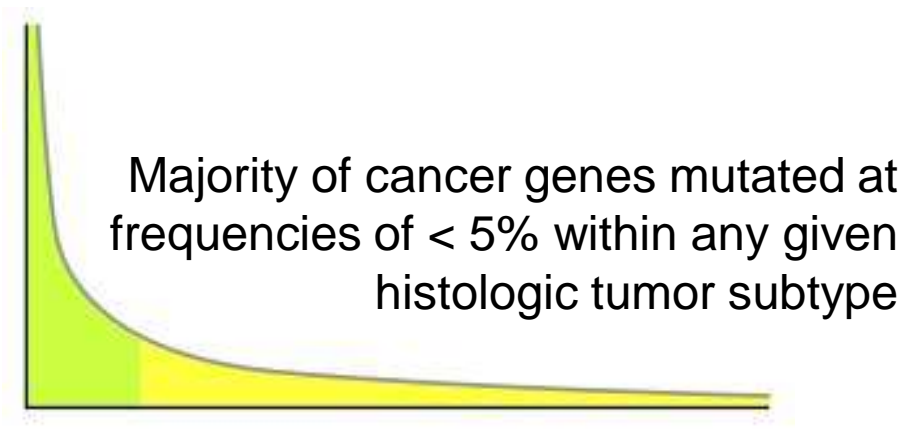

"Long tail" pattern of actionable cancer gene alterations TCGA Pan-Cancer Analysis Lawrence et al. Nature 2013 


\section{NCT MASTER}

\section{Molecularly Aided Stratification for Tumor Eradication Research}

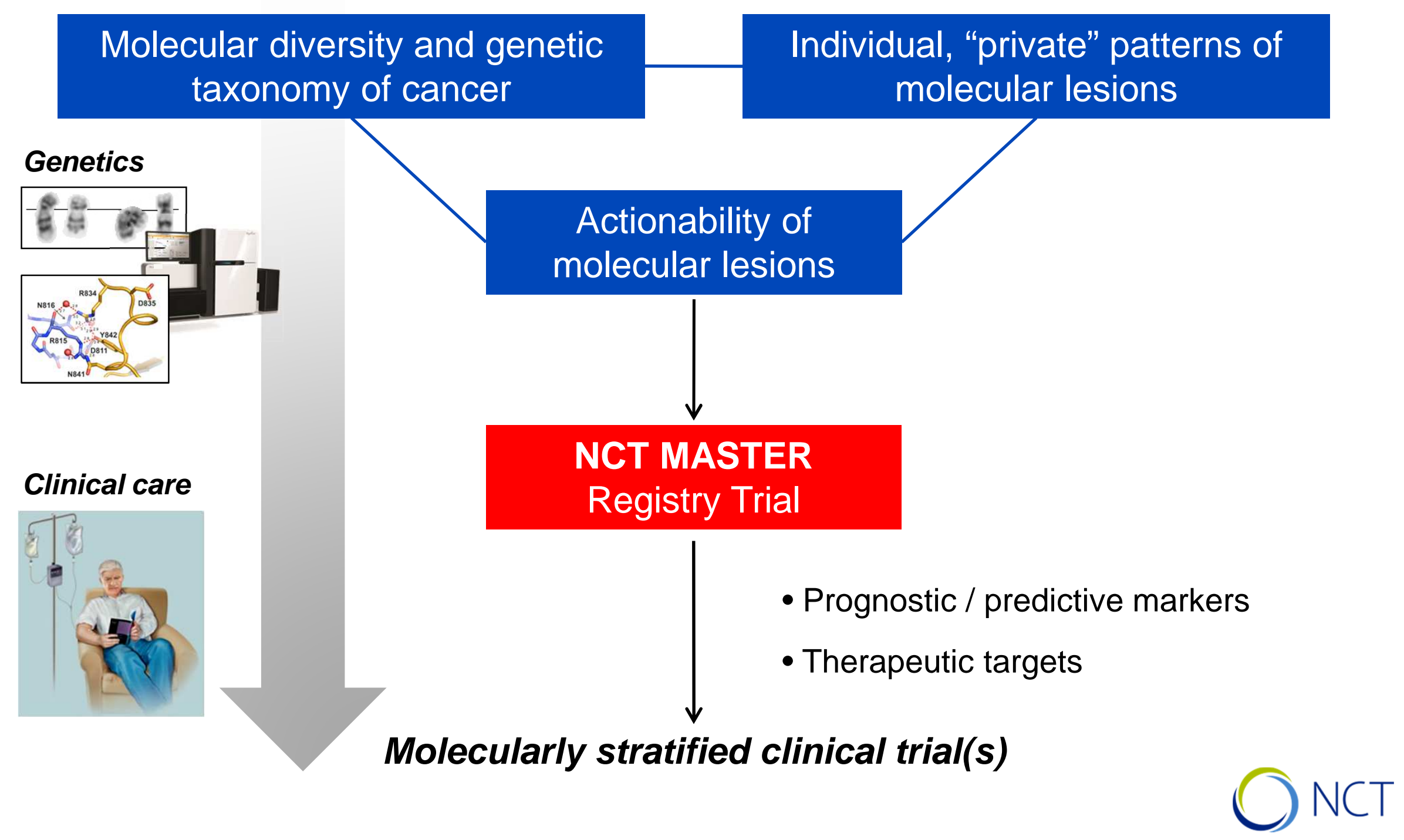




\section{NCT MASTER \\ Eligibility and Objectives}

\section{Eligibility}

- Patients younger than 51 years

- Patients with rare cancers

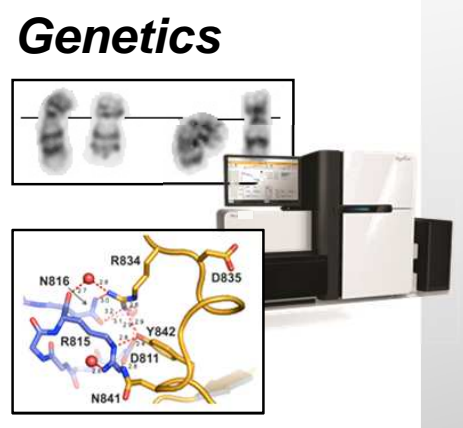

- Incidence of less than 1/100,000 per year

- Measurable disease activity

- No curative treatment available

- Karnofsky Performance Status of at least $70 \%$

- Life expectancy of at least 6 months

\section{Objectives}

\section{Clinical care}

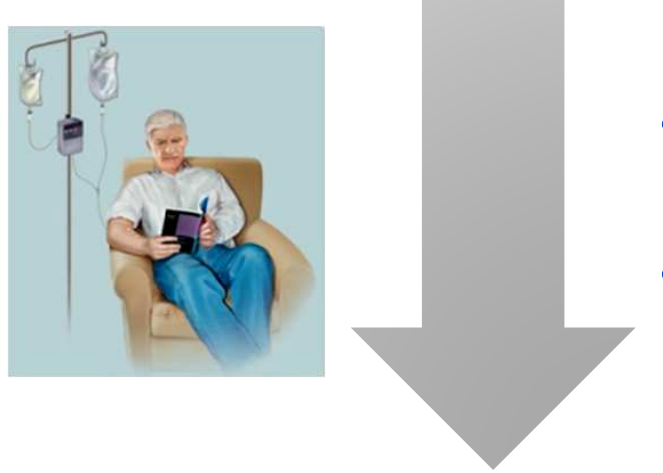

- Prospective exome and transcriptome sequencing within clinical context

- Interdisciplinary evaluation and formulation of treatment recommendations

- Translation into individualized patient care

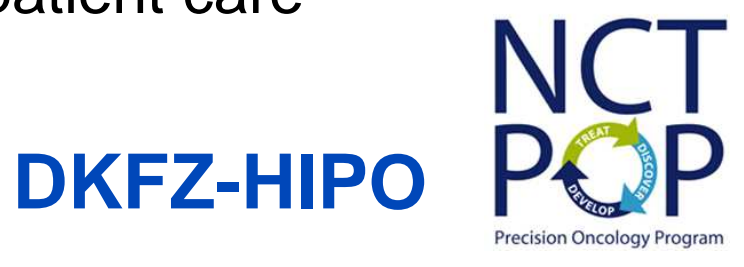




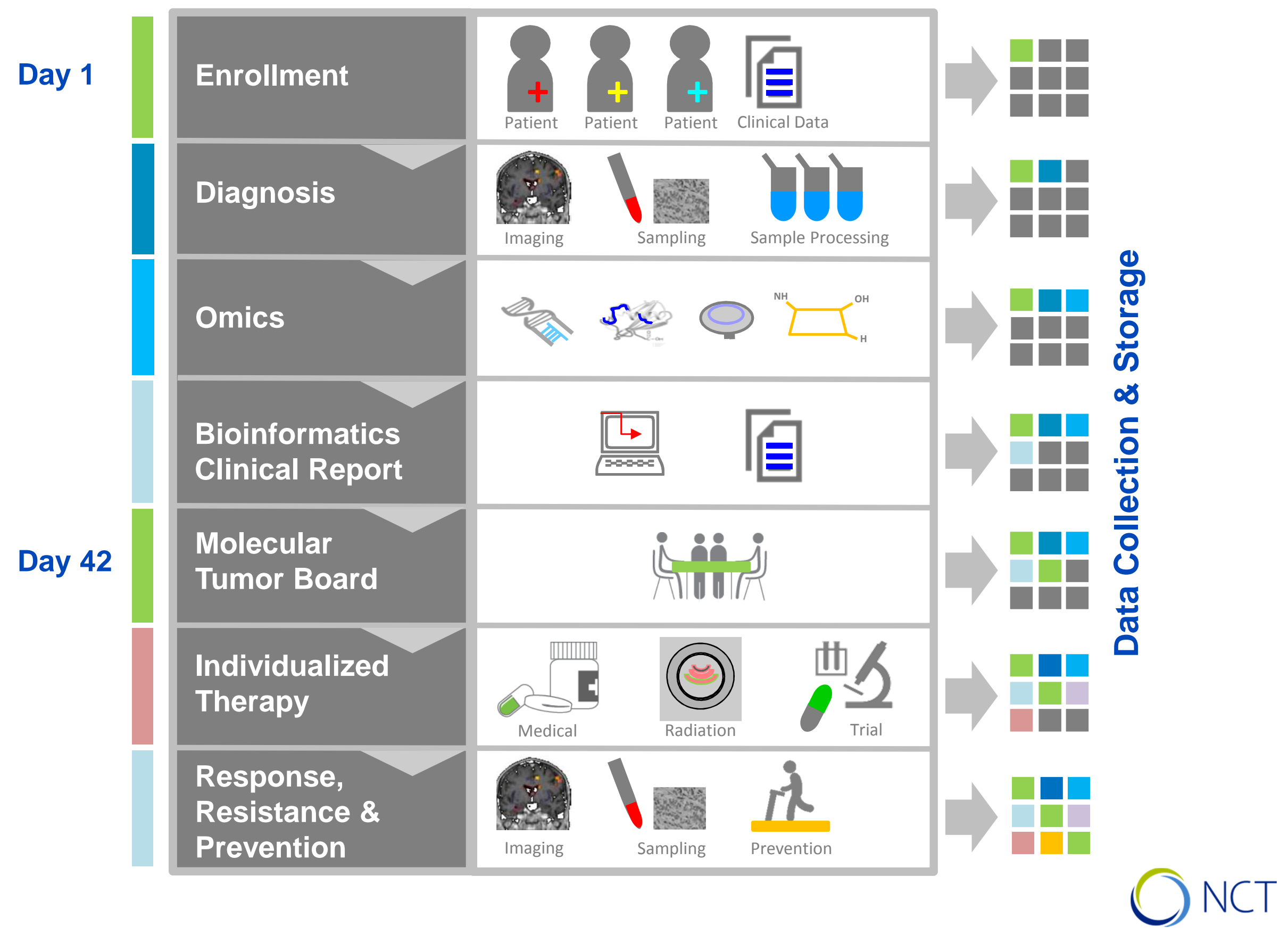




\section{Enrollment NCT MASTER Protocol}

Umbrella protocol for implementing precision oncology at NCT, consenting every patient for:

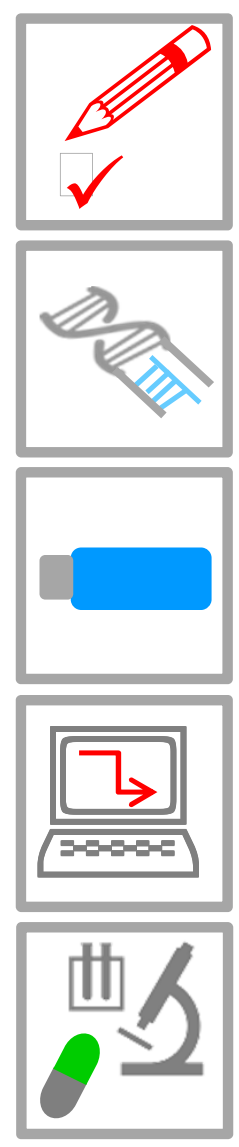

- Questionnaires - Health and Behavior

- Molecular Analysis

- Data Storage

- Clinical Data Analysis

- Recontact for Clinical Trials 


\section{Diagnosis \\ NCT POP / DKFZ-HIPO Sample Processing Lab}

\section{SOP-Guided Preparation of Analytes}

\section{Tasks}

- Sample registration and barcoding

- Sample preparation

- Nucleic acid extraction

- Quality assessment and identity check

- Sample submission to core facility

- Documentation

- Methods development and optimization

- Sample management and storage

\section{Submissions in 2014}

- Exome sequencing

-Whole-genome sequencing

- Transcriptome sequencing

- SNP array profiling

- Expression profiling

- Methylation profiling

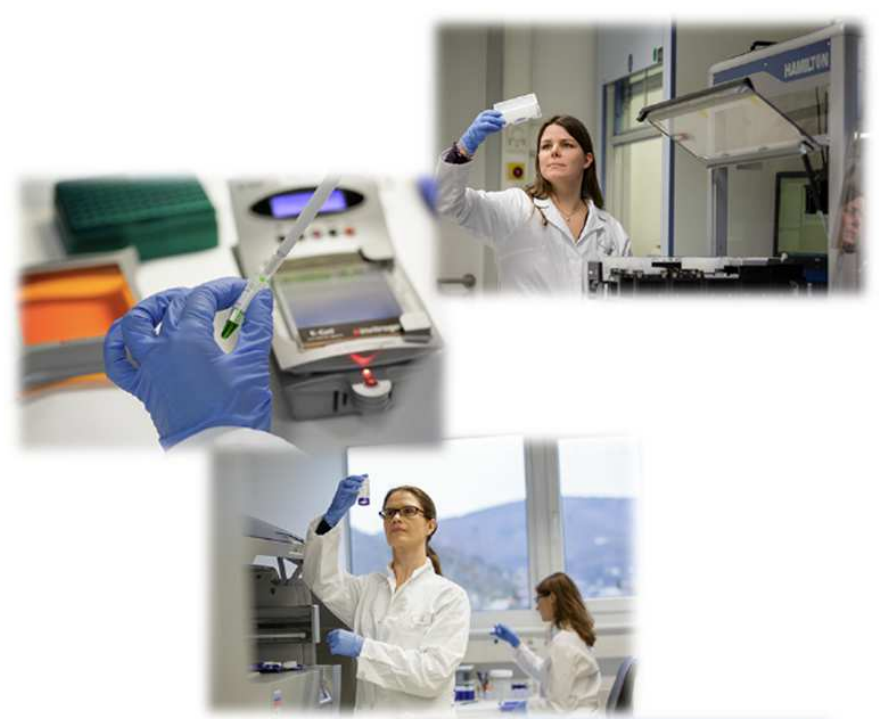

- Total

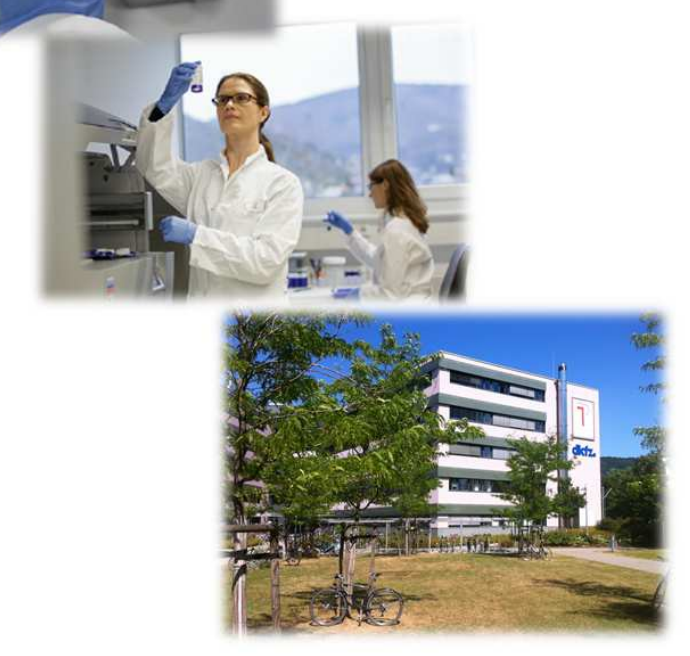

1,932 


\section{Omics, Bioinformatics, Clinical Report}

High-Throughput Sequencing Unit Tasks

- Automated library preparation

- Highly parallel sequencing using Illumina technology

- 14 HiSeq 2000, 2 HiSeq 2500 systems

\section{Clinical Bioinformatics}

\section{Tasks}

- Automated alignment

- Variant calling, selection of somatic mutations

- Gene expression analysis

- Annotation with COSMIC, Cancer Gene Census, potential drugs and pathways

\section{Translational Oncology}

\section{Task}

- Clinical interpretation of molecular data

Molecular Pathology

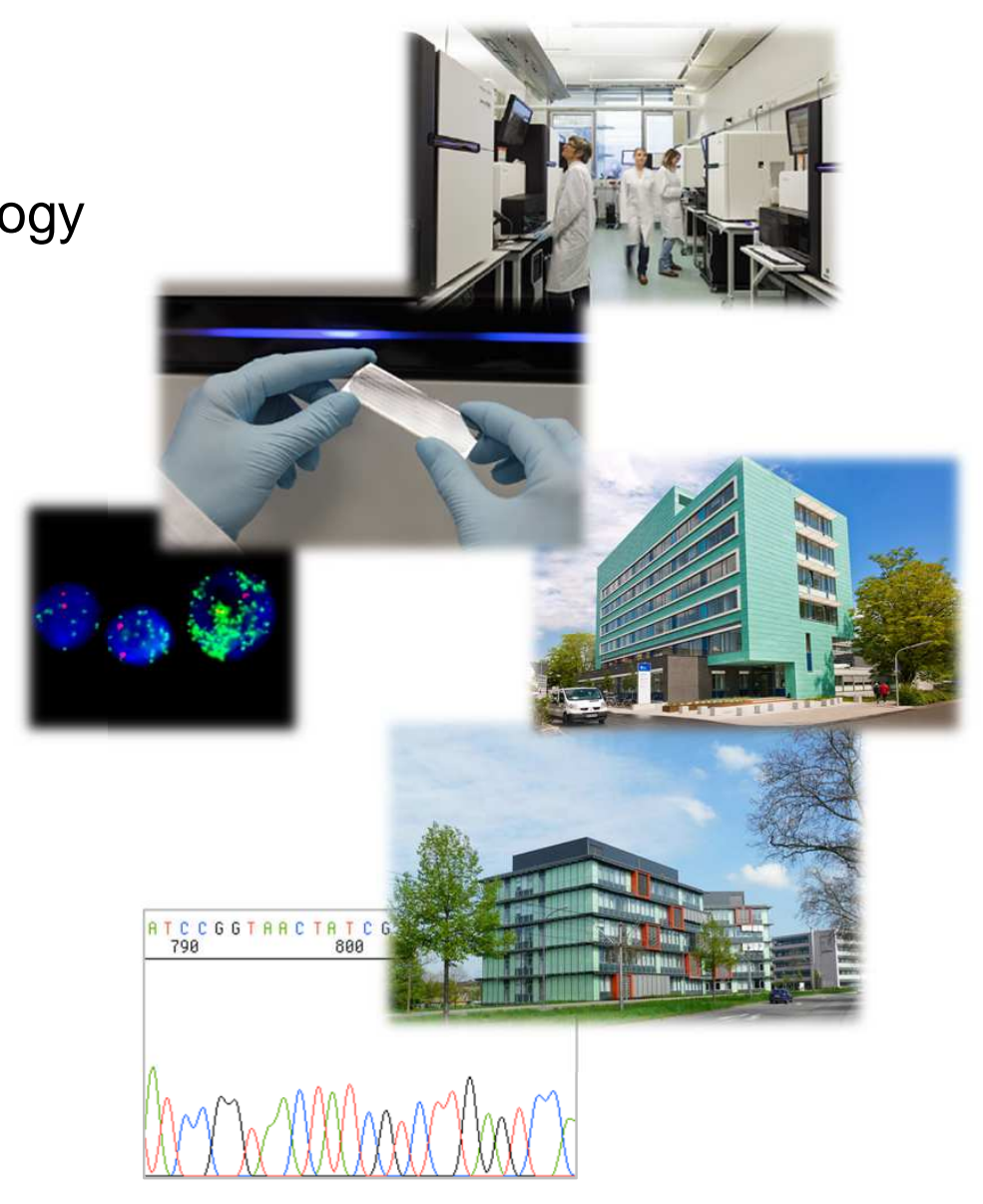

Task

- Validation of actionable genetic alterations 


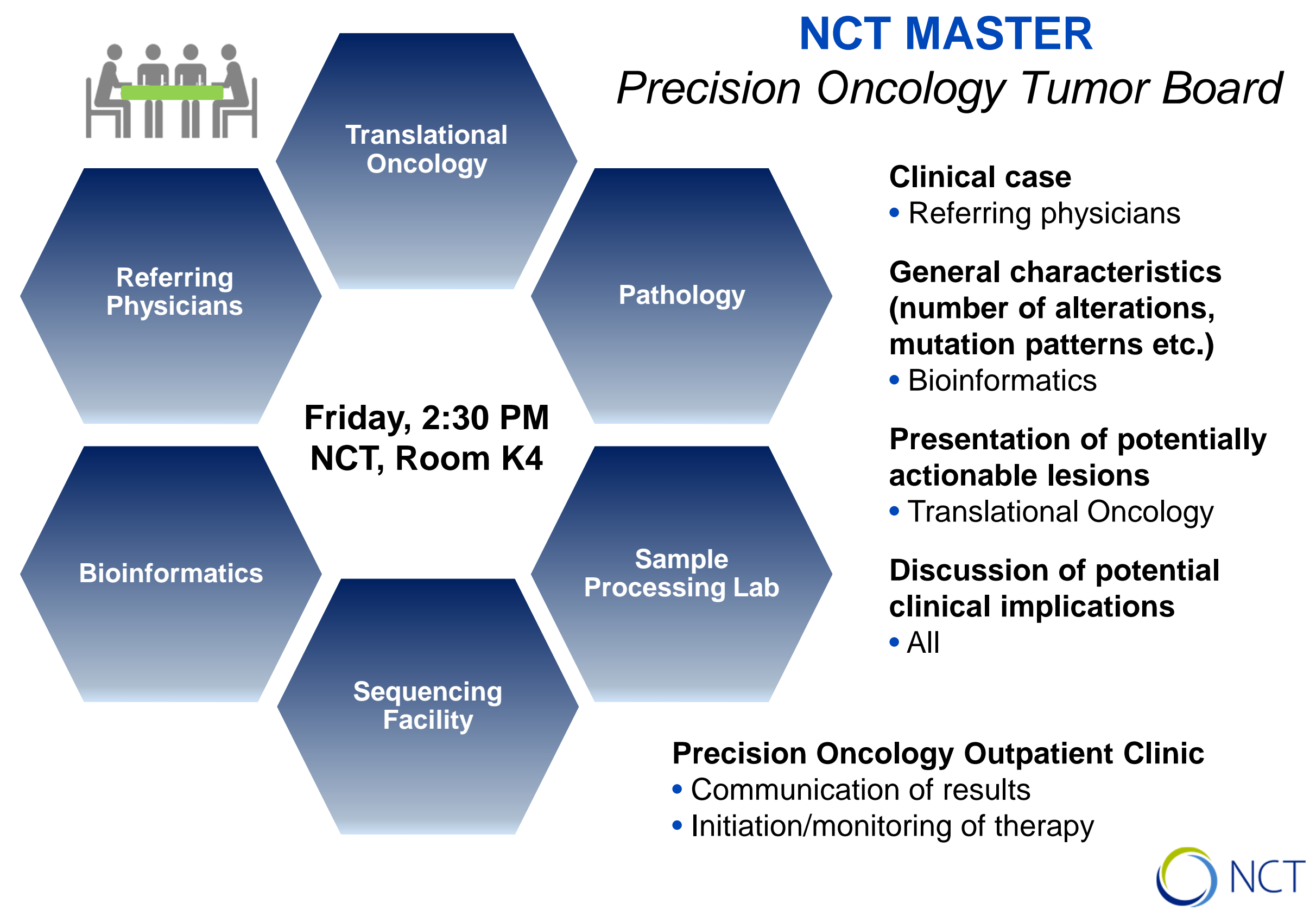




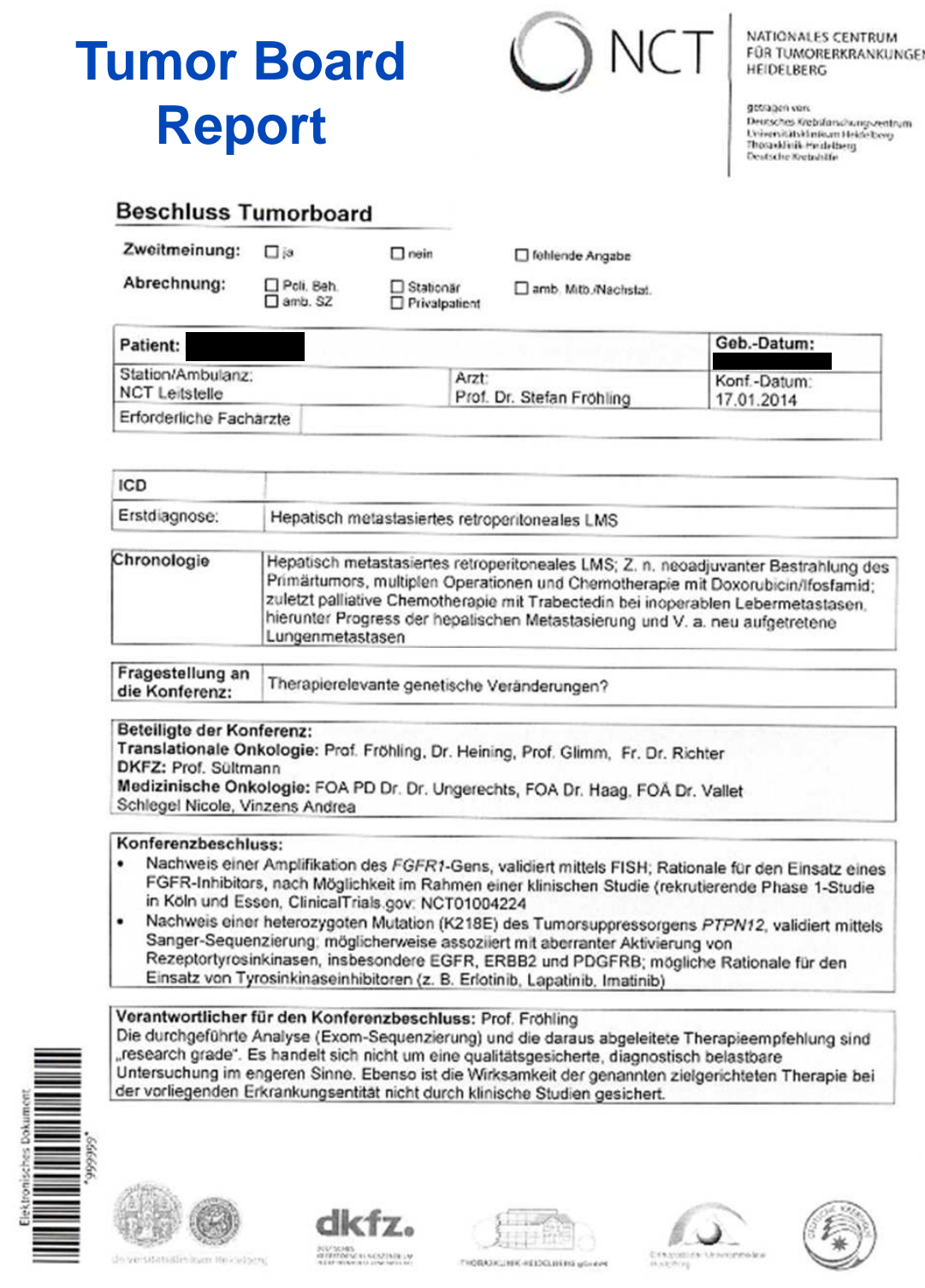

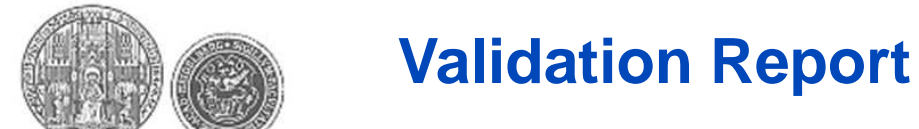

Universitătsklinikum Heidelberg

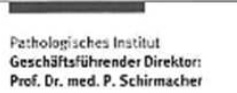

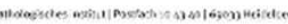

Ergebnis der Mutationsvalidierung im Rahmen des

Personalisierten Onkologie Programms (POP)

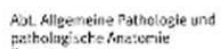
Ärtlicher Direktor:

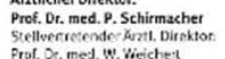

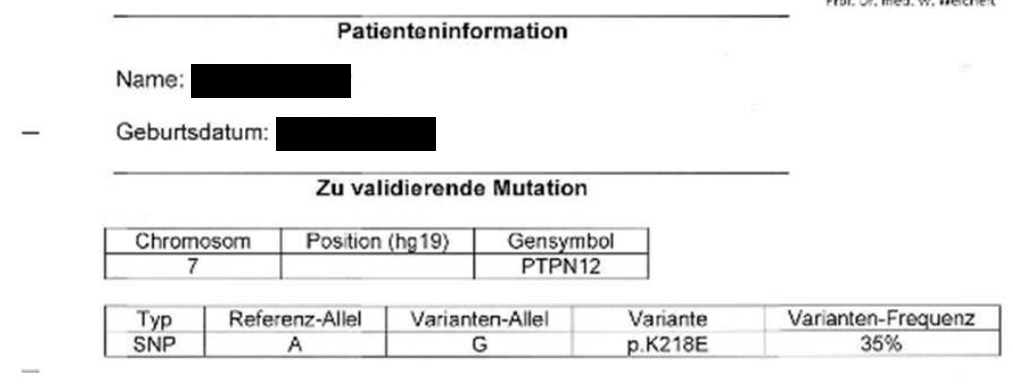

Ergebnis der Sanger-Sequenzierung:

\Die Mutation wurde bestätigt

$\neg$ Die Mutation wurde nicht bestătigt

Bemerkung:

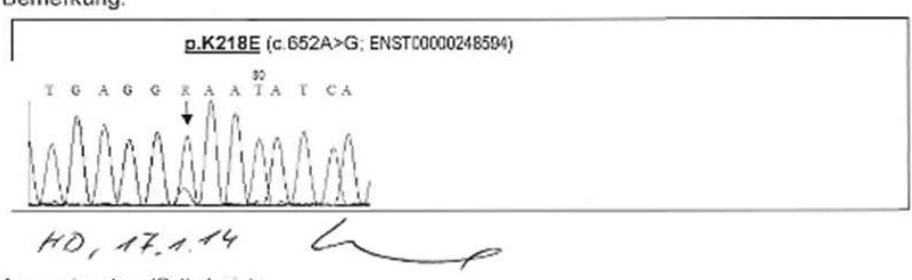

Ansprechpartner (Pathologie):
V. Endris (56-35595); R. Penzel (56-39907)

Die durchgefühtrte Analyse ist ,research grade", es handelt sich nicht urn eine 


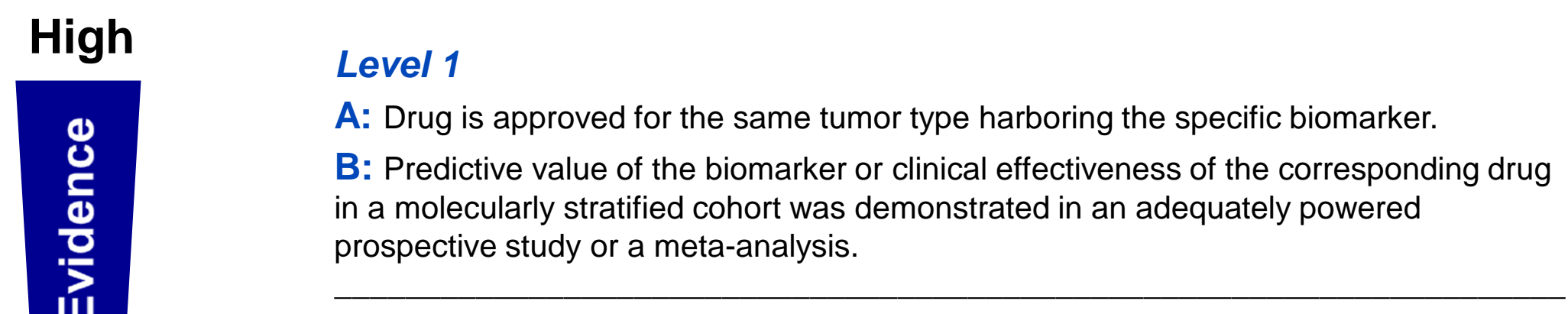

\section{Level 2}

A: Predictive value of the biomarker or clinical effectiveness of the drug in a molecularly stratified cohort was demonstrated in a prospective trial with biomarkers as a secondary objective or an adequately powered retrospective cohort or case-control study in the same tumor type.

B: Predictive value of the biomarker or clinical effectiveness of the drug in a molecularly stratified cohort was demonstrated by clinical data in a different tumor type.

C: Case study or single unusual responder indicates the biomarker is associated with response to the drug, supported by scientific rationale.

\section{Level 3}

Preclinical data (in vitro or in vivo models and functional genomics) demonstrate that the biomarker predicts response of cells to drug treatment.

\section{Level 4}

Biological rationale exists that links the drug to the altered signaling pathway or relevant basket. No reported clinical or preclinical data on the response to the drug. 


\section{NCT MASTER}

\section{Current Status}

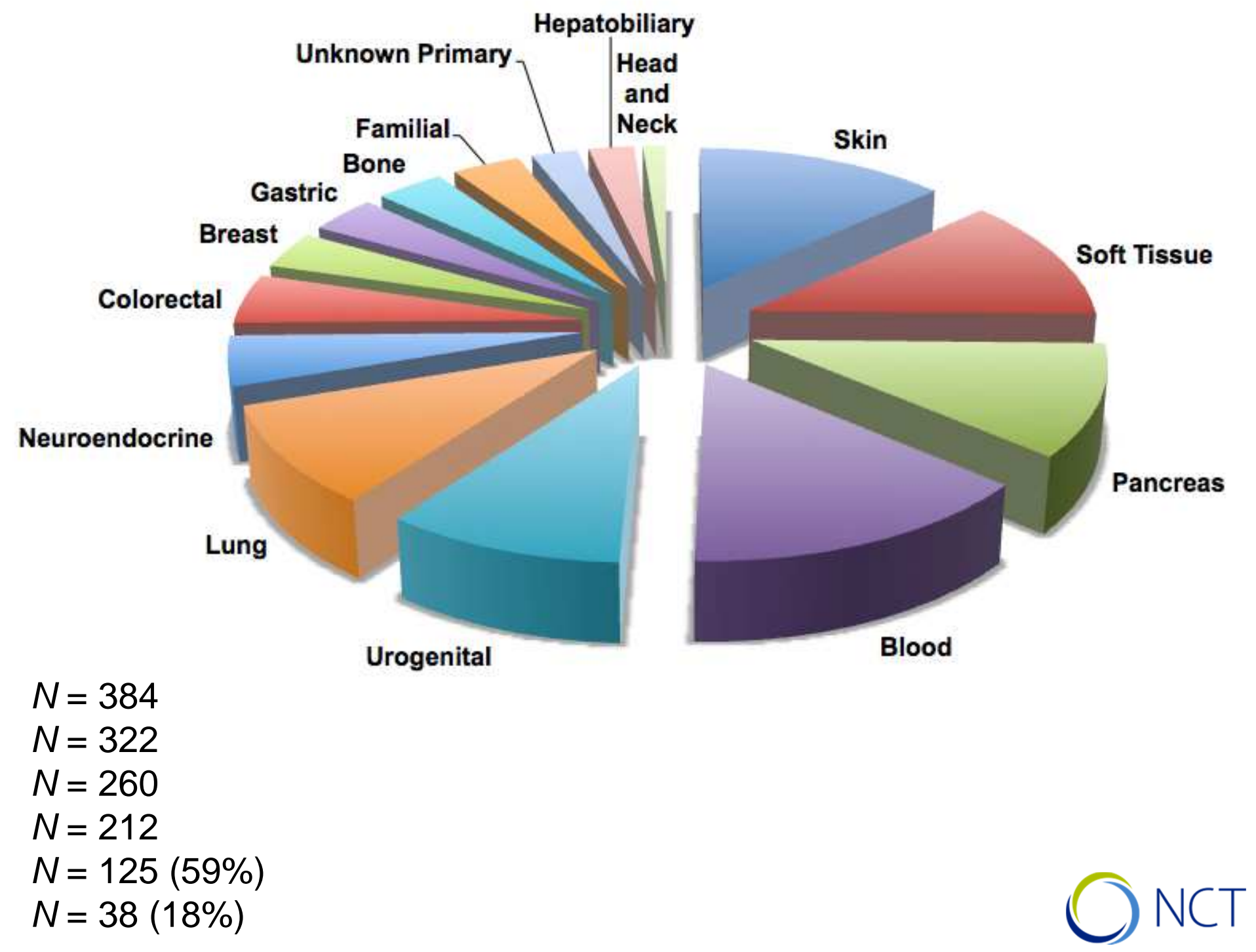

July 2015

Registered

Eligible

Completed

Discussed

Recommendation

Treatment
$N=384$

$N=322$

$N=260$

$N=212$

$N=38(18 \%)$ 


\section{NCT MOLECULAR DIAGNOSTICS PROGRAM Personalized Oncology at NCT - NCT MASTER}

\section{Findings With Established Clinical Implications by Histology}

\begin{tabular}{lll}
\hline Disease & Mutation & Clinical Action \\
\hline Melanoma & BRAF p.V600R & Vemurafenib, dabrafenib \\
& ERBB4 p.P172F & Lapatinib \\
\hline Uveal melanoma & GNAQ p.Q209L/P/R & Trametinib, selumetinib \\
\hline Basal-cell carcinoma & PTCH1 p.648_650del & Vismodegib \\
Multiple myeloma & BRAF p.V600E & Vemurafenib, dabrafenib \\
\hline Myxoid liposarcoma & PIK3CA p.C420R/p.E545K & PI3K/AKT inhibitors, \\
& PTEN p.R130G & everolimus, temsirolimus \\
Gastrointestinal stromal tumor & KIT p.V560D & Imatinib \\
& PDGFRA p.D842V & Crenolanib \\
\hline Ovarian cancer & TSC2 p.R505X & Everolimus, temsirolimus \\
Breast cancer & PIK3CA p.E545K & PI3K/AKT inhibitors, \\
& TSC1/2del & everolimus, temsirolimus \\
Pulmonary adenocarcinoma & FGFR1 amp & FGFR inhibitors \\
\hline Carcinoma of unknown primary & EGFR p.T790M & AZD9291 \\
& TSC2mut & Everolimus, temsirolimus \\
\hline
\end{tabular}




\section{NCT MOLECULAR DIAGNOSTICS PROGRAM Personalized Oncology at NCT - NCT MASTER}

Findings With Established Clinical Implications by Gene/Pathway

\begin{tabular}{|c|c|c|}
\hline Mutation & Disease & Clinical Action \\
\hline BRAF p.V600R/p.V600E & $\begin{array}{l}\text { Multiple myeloma } \\
\text { Melanoma }\end{array}$ & Vemurafenib, dabrafenib \\
\hline PIK3CA p.C420R/p.E545K & $\begin{array}{l}\text { Breast cancer } \\
\text { Myxoid liposarcoma } \\
\text { Carcinoma of unknown primary }\end{array}$ & $\begin{array}{l}\text { PI3K/AKT inhibitors, } \\
\text { everolimus, temsirolimus }\end{array}$ \\
\hline $\begin{array}{l}\text { TSC1 }{ }^{\text {mut} / T S C 1 ~} 1^{\text {del }} \\
\text { TSC2 }^{\text {mut}} /{ }^{\text {TSC }} 2^{\text {del }}\end{array}$ & $\begin{array}{l}\text { Esophageal adenocarcinoma } \\
\text { Gastric cancer } \\
\text { Breast cancer } \\
\text { Ovarian cancer } \\
\text { Pulmonary adenocarcinoma }\end{array}$ & Everolimus, temsirolimus \\
\hline FGFR 1 amp & $\begin{array}{l}\text { Breast cancer } \\
\text { Leiomyosarcoma } \\
\text { T-cell prolymphocytic leukemia }\end{array}$ & FGFR inhibitors \\
\hline KIT p.V560D/p.D579del & $\begin{array}{l}\text { Gastrointestinal stromal tumor } \\
\text { Sinonasal undifferentiated } \\
\text { carcinoma }\end{array}$ & Imatinib \\
\hline ERBB2amp/p.D769Y & $\begin{array}{l}\text { Colorectal cancer } \\
\text { Cholangiocarcinoma } \\
\text { Gallbladder carcinoma } \\
\text { Plexiform schwannoma }\end{array}$ & $\begin{array}{l}\text { Trastuzumab, pertuzumab, } \\
\text { lapatinib, neratinib }\end{array}$ \\
\hline
\end{tabular}




\section{NCT MOLECULAR DIAGNOSTICS PROGRAM Personalized Oncology at NCT - NCT MASTER}

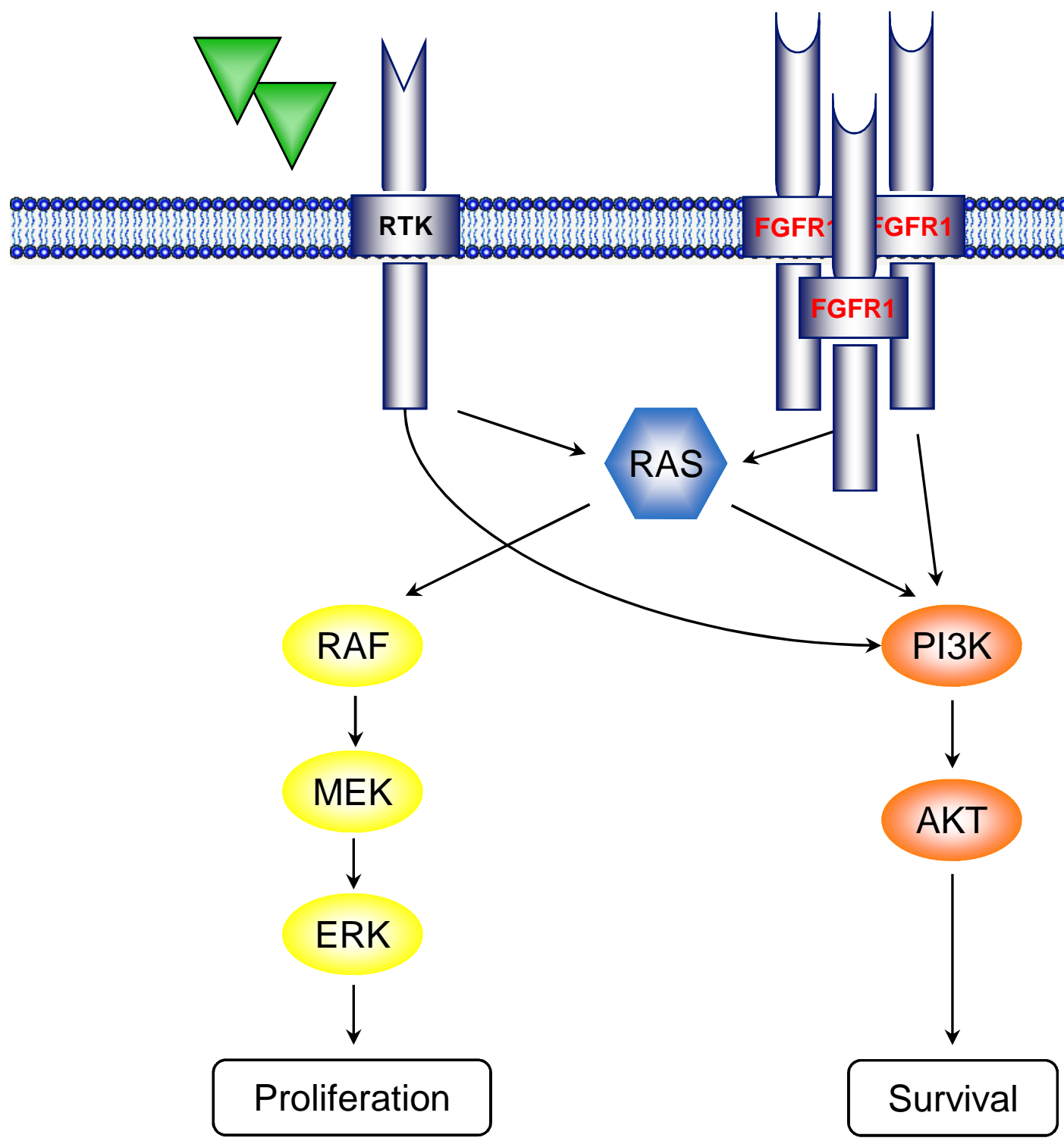

\section{Rationale for Experimental Targeted Treatment (1)}

retroperitoneal leiomyosarcoma

- No local therapy options

- Refractory to doxorubicin/ifosfamide

- Refractory to trabectedin

Focal 8p amplification involving FGFR1, validated by FISH

Treatment with FGFR inhibitor within phase 1 clinical trial for 2 months

- Discontinuation due to adverse events

Ongoing stable disease for $>12$ months with minimal metabolic activity of metastatic lesions

Proliferation

Survival 


\section{NCT MOLECULAR DIAGNOSTICS PROGRAM Personalized Oncology at NCT - NCT MASTER}

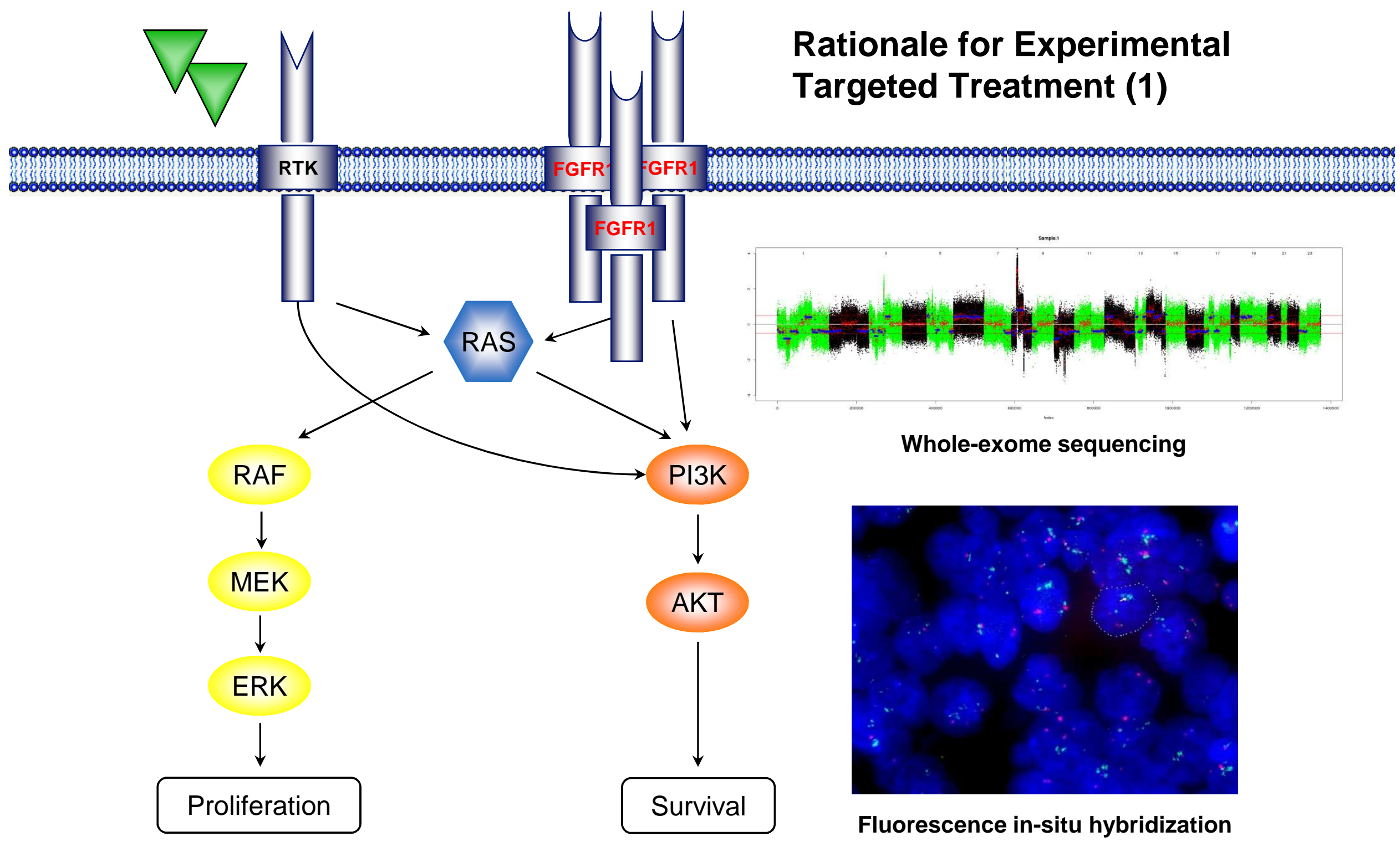




\section{NCT MOLECULAR DIAGNOSTICS PROGRAM Personalized Oncology at NCT - NCT MASTER}

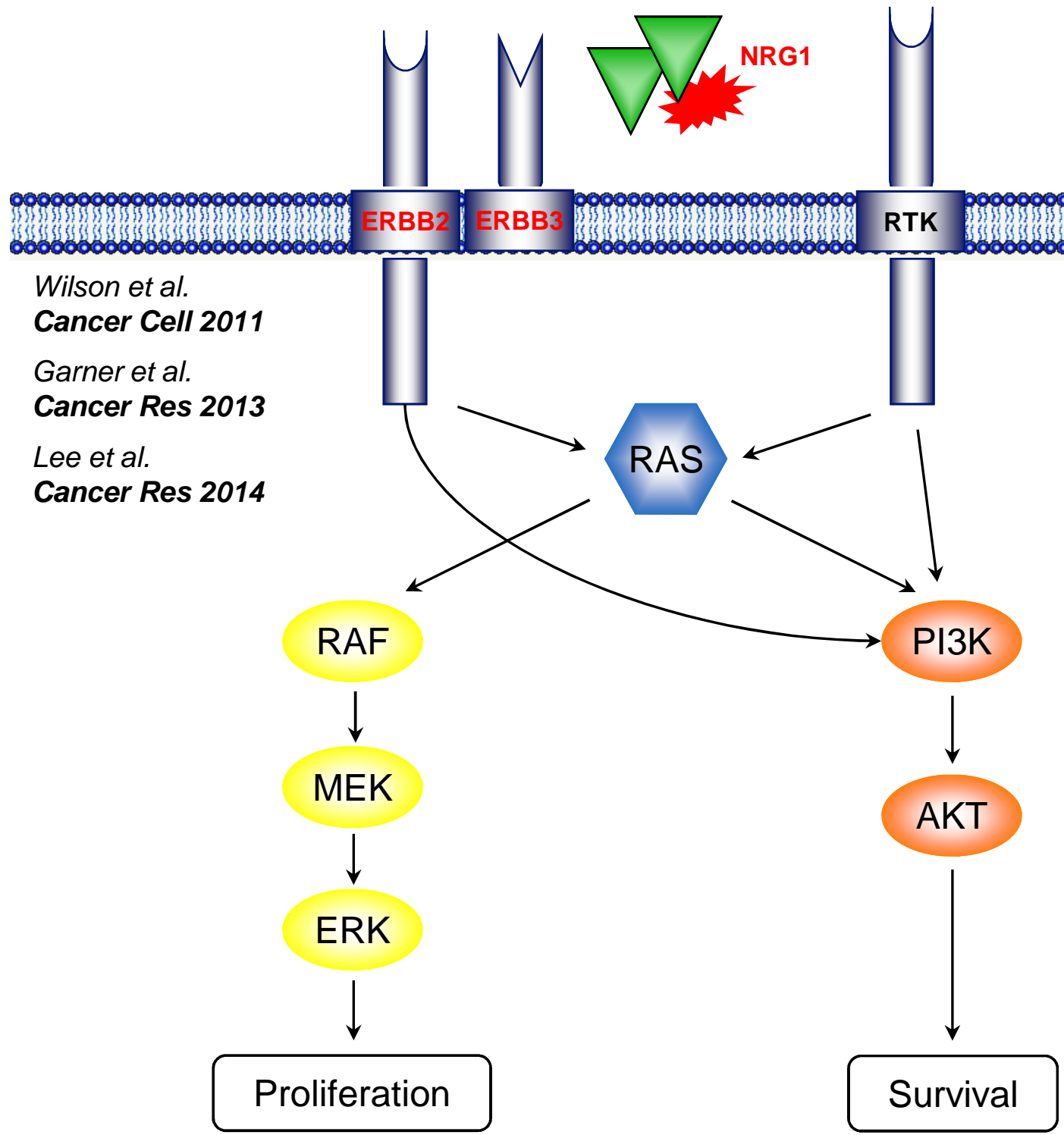

\section{Rationale for Experimental Targeted Treatment (2)}

46-year-old patient with metastatic triplenegative breast cancer

- Refractory to multiple lines of chemotherapy

NRG1 c.1053-1G>A (splice site mutation) NRG1 expression by immunohistochemistry

Aberrant NRG1 expression and ERBB3 activation, enhanced dimerization with other ERBB family members, and constitutive signal transduction as rationale for pan-ERBB blockade in "ERBB2-low" breast cancer

Treatment with pertuzumab, eribulin, and bevacizumab

Stable disease for $>6$ months

Proliferation

Survival 


\section{NCT MOLECULAR DIAGNOSTICS PROGRAM Personalized Oncology at NCT - NCT MASTER}

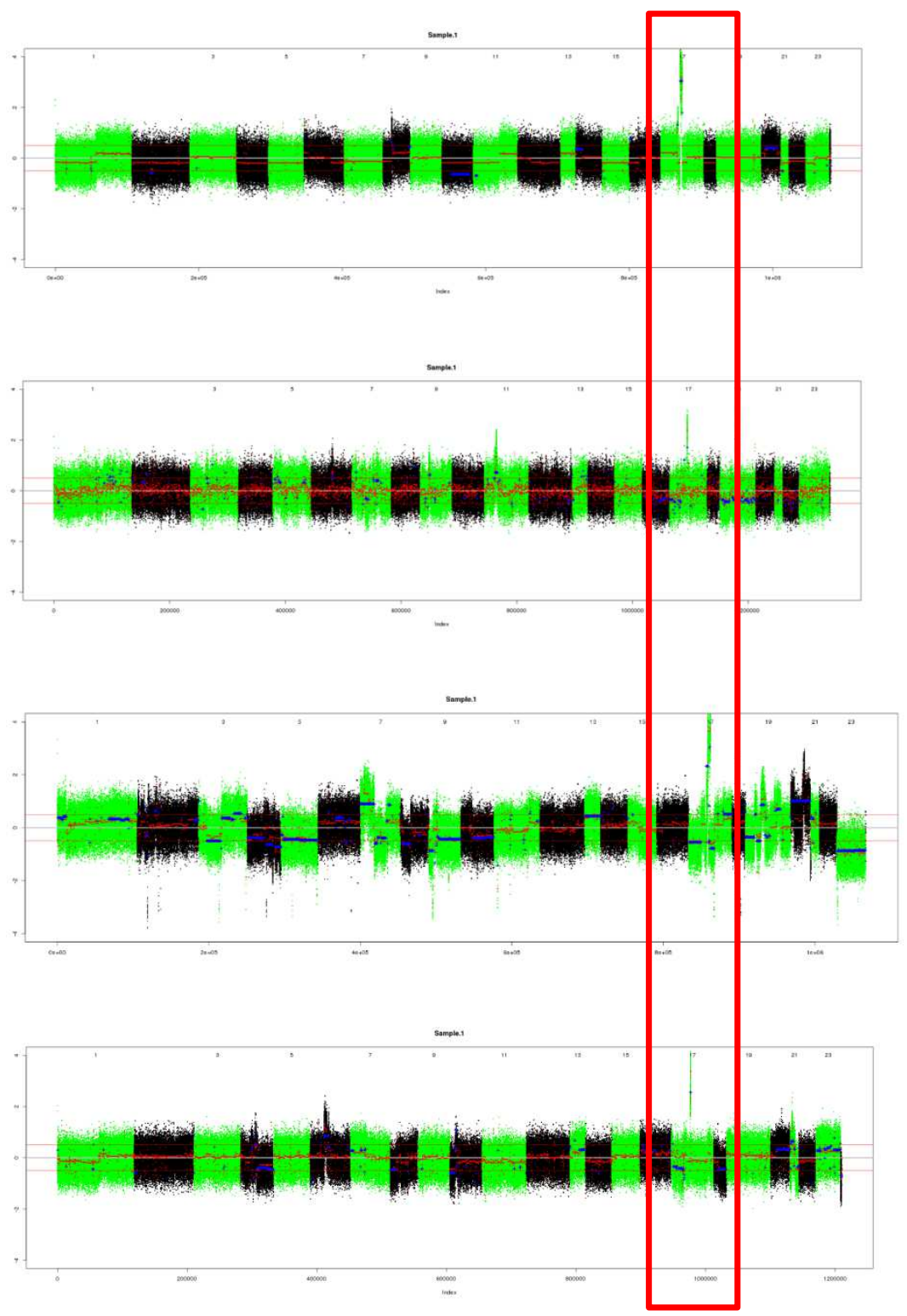

\section{Rationale for Experimental Targeted Treatment (3)}

CRC

ERBB2 amplification and overexpression in gastrointestinal cancers

- KRASWT colorectal cancer $(n=3)$

- Gallbladder carcinoma $(n=1)$

- Cholangiocarcinoma $(n=1)$

$\mathrm{GBC}^{*}$

Aberrant ERBB2 expression and constitutive signal transduction as rationale for ERBB2 blockade with trastuzumab, pertuzumab, or lapatinib

CCC

*Ongoing partial remission in a 37-year-old patient with metastatic gallbladder carcinoma treated with trastuzumab, pertuzumab, and nab-paclitaxel since 11/2014

\section{HERACLES Trial}

Trastuzumab/lapatinib in patients with heavily pretreated, ERBB2 ${ }^{\mathrm{amp}}$, KRASWT colorectal cancer; DCR: 78\%; median TTP: 5.5 months Siena et al. ASCO Annual Meeting 2015 


\section{NCT MOLECULAR DIAGNOSTICS PROGRAM Personalized Oncology at NCT - NCT MASTER}

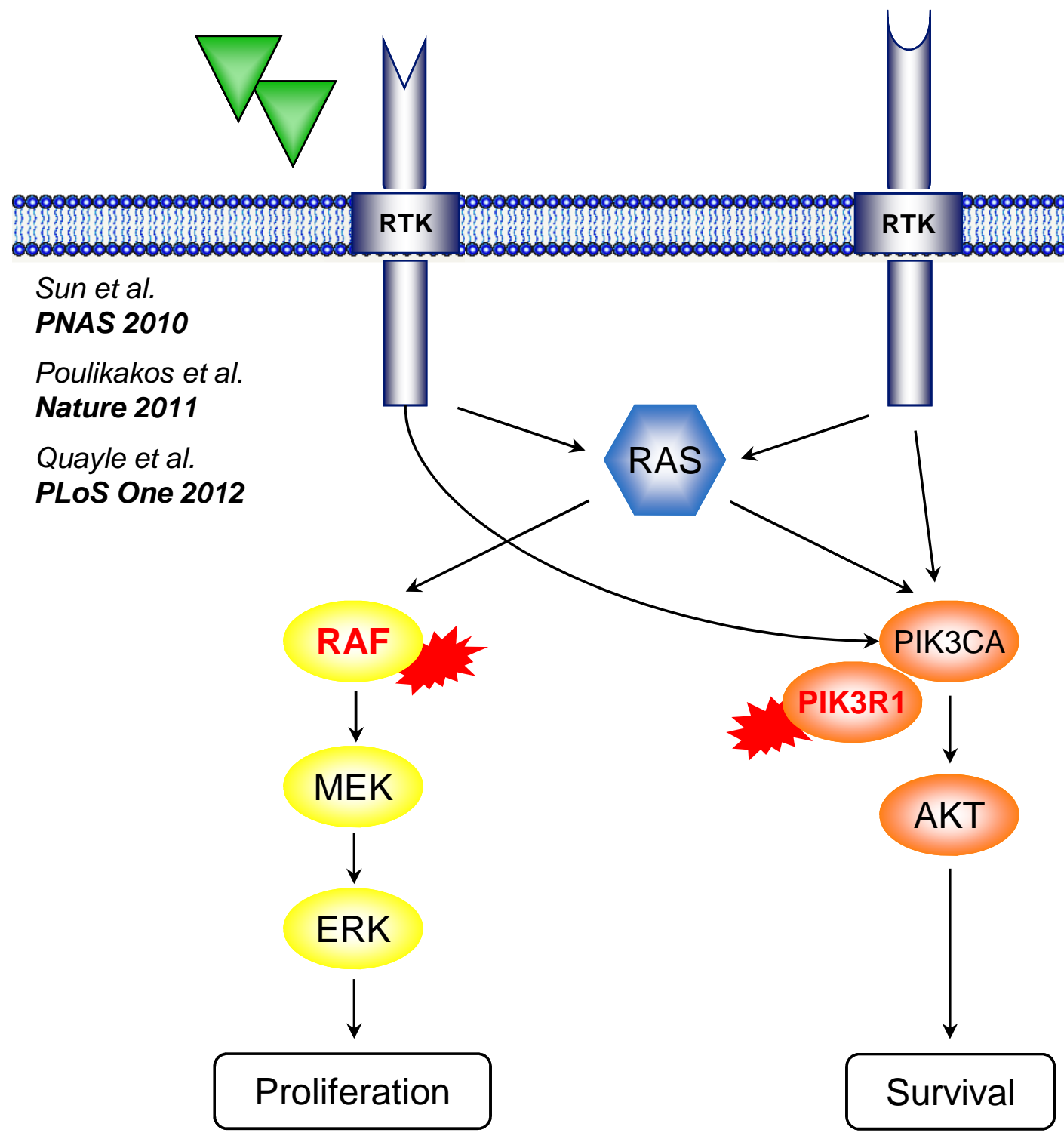

Rationale for Experimental Targeted Treatment (6)

66-year-old patient with glioblastoma

- Status post incomplete resection

- Adjuvant chemotherapy with temozolomide

PIK3R1 p.G376R

- Activation of PI3K, low transforming potential

TTYH3-BRAF fusion

- Novel, structurally distinct fusion involving near-full-length BRAF

- BRAF activation and transforming potential

\section{BRAF p.47_438del}

- Loss of N-terminal regulatory domain and constitutive BRAF activation

- Described as splicing alteration associated with constitutive BRAF dimerization and resistance to vemurafenib in melanoma

Rationale for MAPK pathway blockade using sorafenib or MEK inhibitor 


\section{NCT MOLECULAR DIAGNOSTICS PROGRAM}

\section{Personalized Oncology at NCT - NCT MASTER}

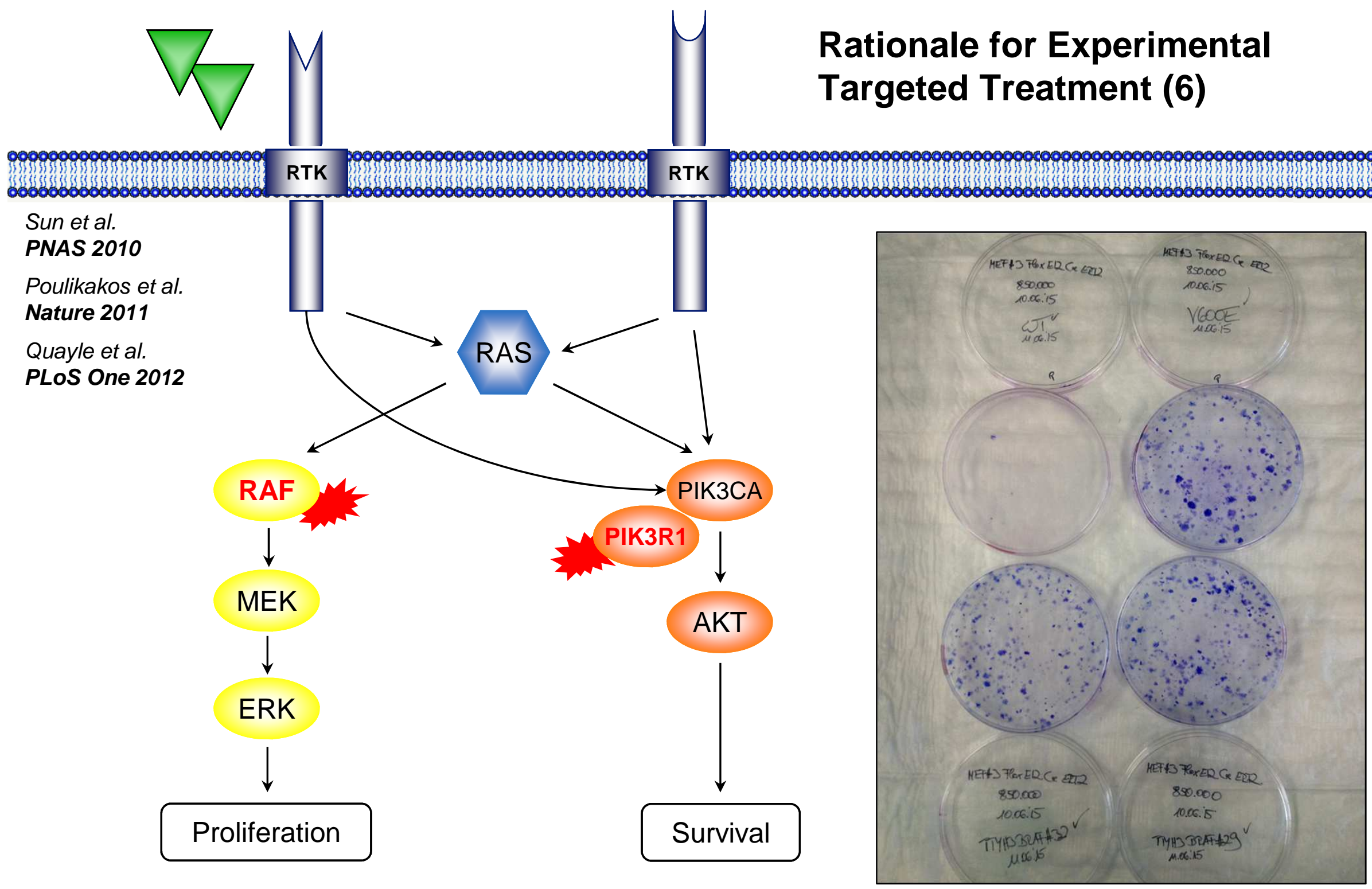




\section{NCT MOLECULAR DIAGNOSTICS PROGRAM}

\section{Personalized Oncology at NCT - NCT MASTER}

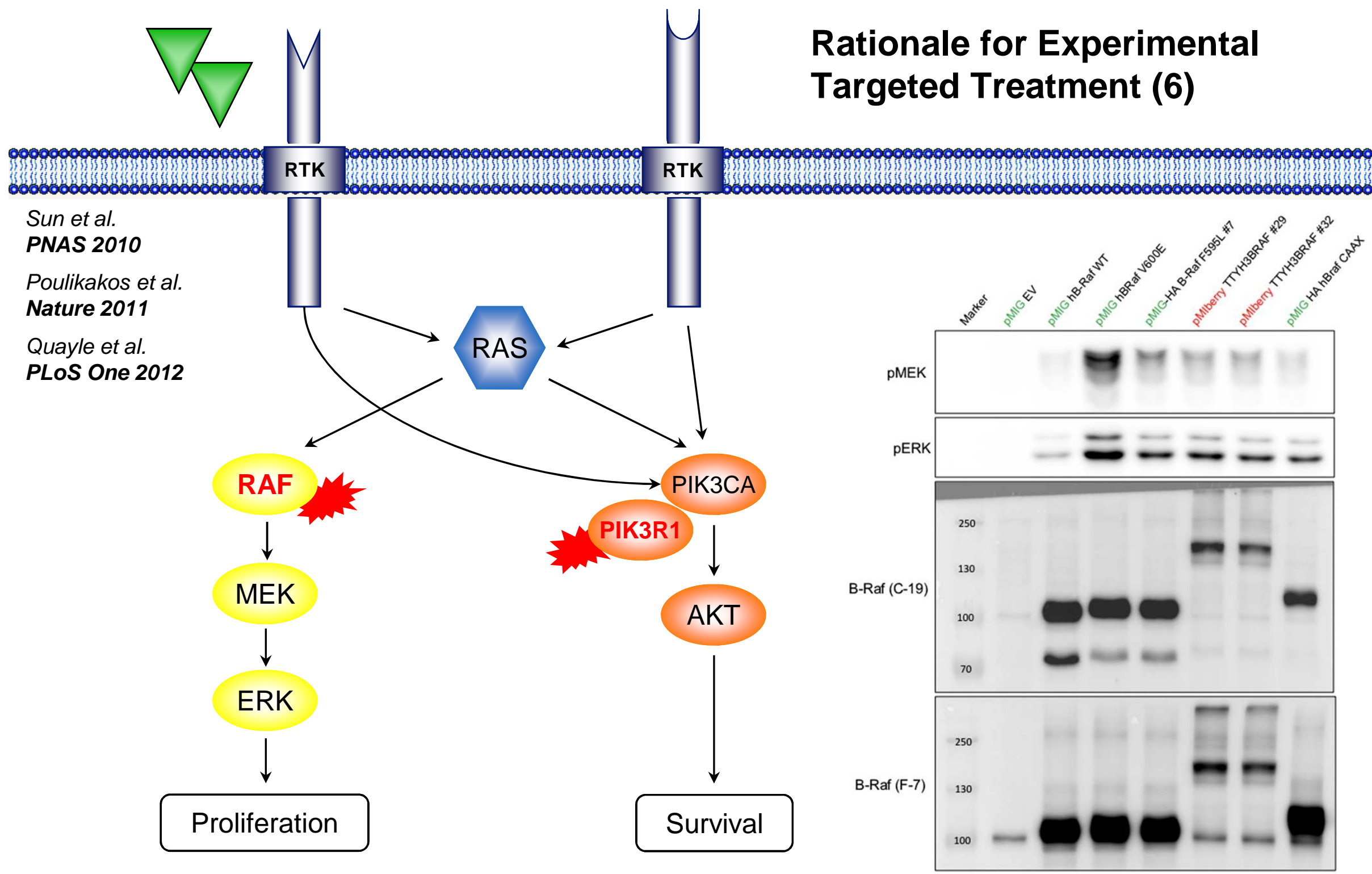




\section{NCT MOLECULAR DIAGNOSTICS PROGRAM Personalized Oncology at NCT - NCT MASTER}

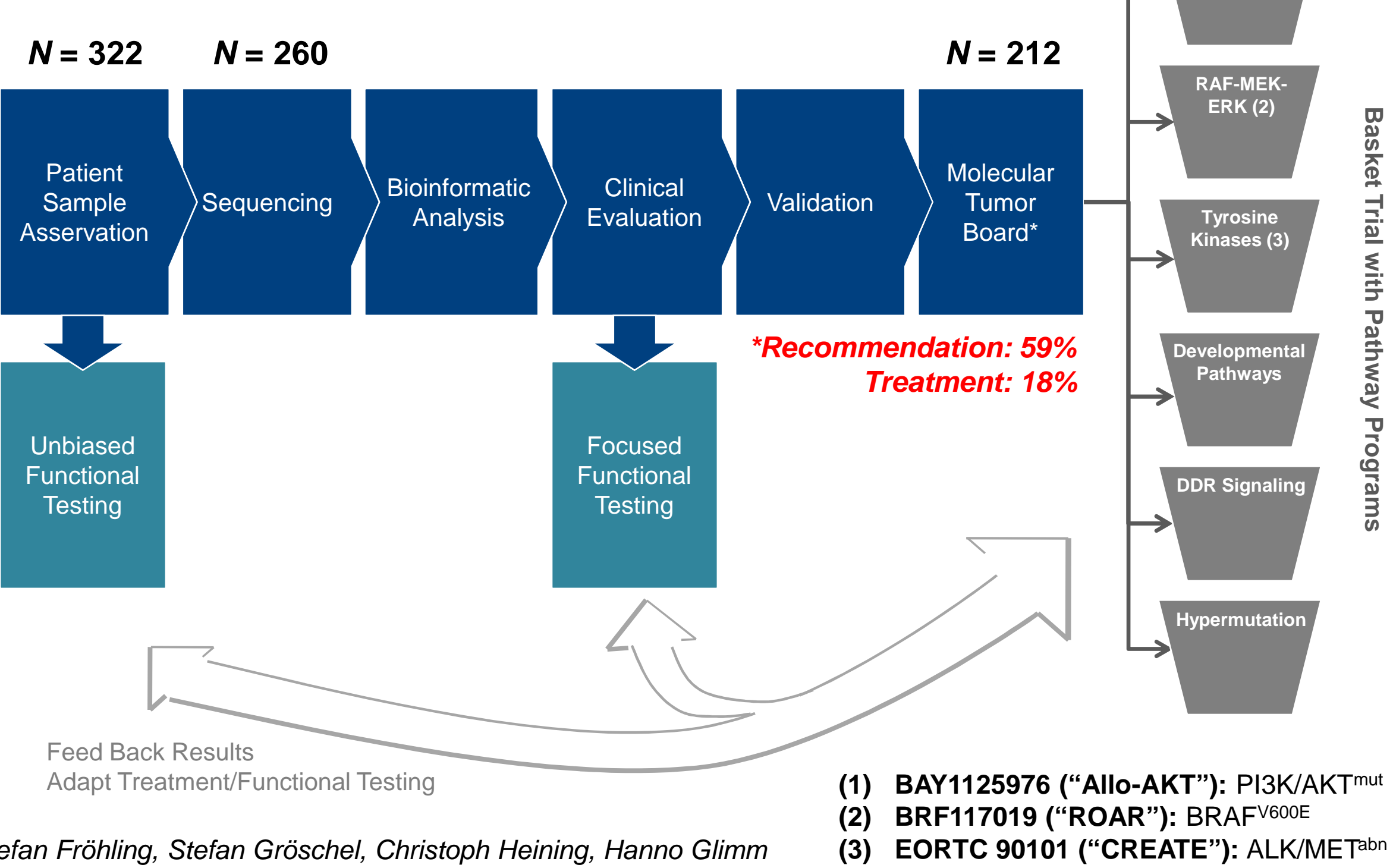

Claudia Scholl (Functional Genomics)

(3) EORTC 90101 (“CREATE”): ALK/METabn

DKTK: Frankfurt/Mainz, Munich, Dresden (Berlin, Essen, Freiburg) 


\section{MOLECULAR STRATIFICATION PROGRAMS}

Mi-Oncoseq vs. UCSD PREDICT vs. NCT MASTER

\begin{tabular}{|c|c|c|c|c|c|}
\hline Program & Analyses & Patients & Actionable & Action & Outcome \\
\hline Mi-Oncoseq & $\begin{array}{c}\text { WES } \\
\text { RNA-seq }\end{array}$ & 369 & $59 \%$ & $23 \%$ & Response \\
\hline $\begin{array}{c}\text { UCSD } \\
\text { PREDICT }\end{array}$ & $\begin{array}{c}\text { Panel-seq } \\
(236 \text { genes })\end{array}$ & 347 & - & $25 \%$ & $\begin{array}{c}\text { Response, } \\
\text { PFS, OS }\end{array}$ \\
\hline $\begin{array}{c}\text { NCT } \\
\text { MASTER }\end{array}$ & $\begin{array}{c}\text { WES } \\
\text { RNA-seq }\end{array}$ & 212 & $59 \%$ & $18 \%$ & Response \\
\hline
\end{tabular}

${ }^{1}$ Rationale for drug in development or off-label use of approved drug; prediction of response to treatment; predisposing germline alteration

${ }^{2}$ SD/PR/CR: 35\% vs. $16 \%$ ( $\left.P=0.02\right)$; PFS: 4 vs. 3 months ( $P=0.04$ ); PFS2/PFS1 $\geq 1,3: 45 \%$ vs. $19 \%(P=0.004$ and $P=0.06)$; trend for superior PFS following treatment "matched directly" $v s$. "matched indirectly" $(P=0.1)$; OS: 14 vs. 11 months (NS) 


\section{LONG-TERM OUTCOME OF STRATIFIED THERAPY \\ Meta-Analysis of Targeted Treatment Strategies}

- UC San Diego, MD Anderson Cancer Center, Institut Gustave Roussy, WIN Consortium, ASCO

- $\quad 570$ phase 2 trials; 32,149 patients; targeted agents as monotherapy; 641 treatment arms

- Therapy according to biomarker vs. unselected treatment

- Superior outcome compared to conventional chemotherapy through targeted therapy according to biomarker ("matched directly" and "matched indirectly")

- Response, PFS, OS (each $P<0.0001$ )

- Toxicity $(P<0.001)$

- Inferior outcome compared to conventional chemotherapy through unselected "targeted" therapy

- Response, PFS (each $P<0.0001$ )

- OS $(P=0.048)$

- Better outcome with targeted approaches based on genomic alterations (mutations) vs. aberrant expression (RNA, protein) as biomarker

- Response, PFS, OS (each $P<0.05$ ) 


\section{NCT MOLECULAR DIAGNOSTICS PROGRAM Personalized Oncology at NCT - NCT MASTER}

$N=322 \quad N=260$
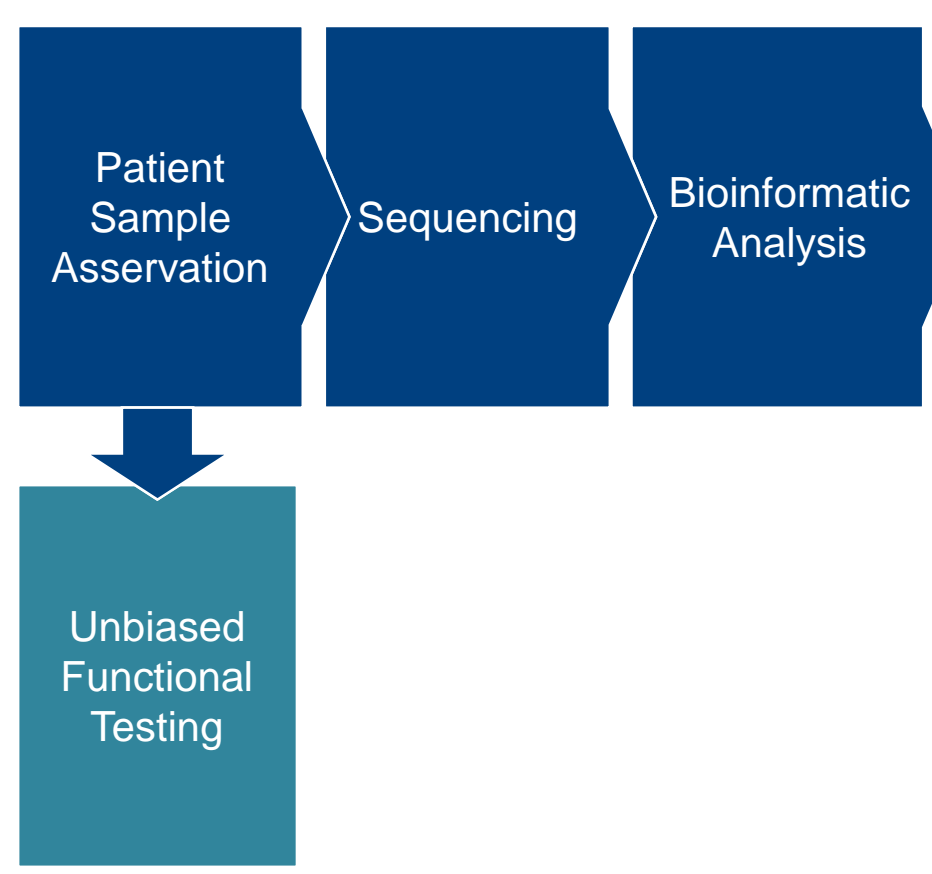

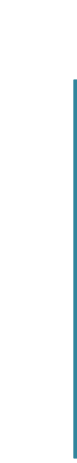

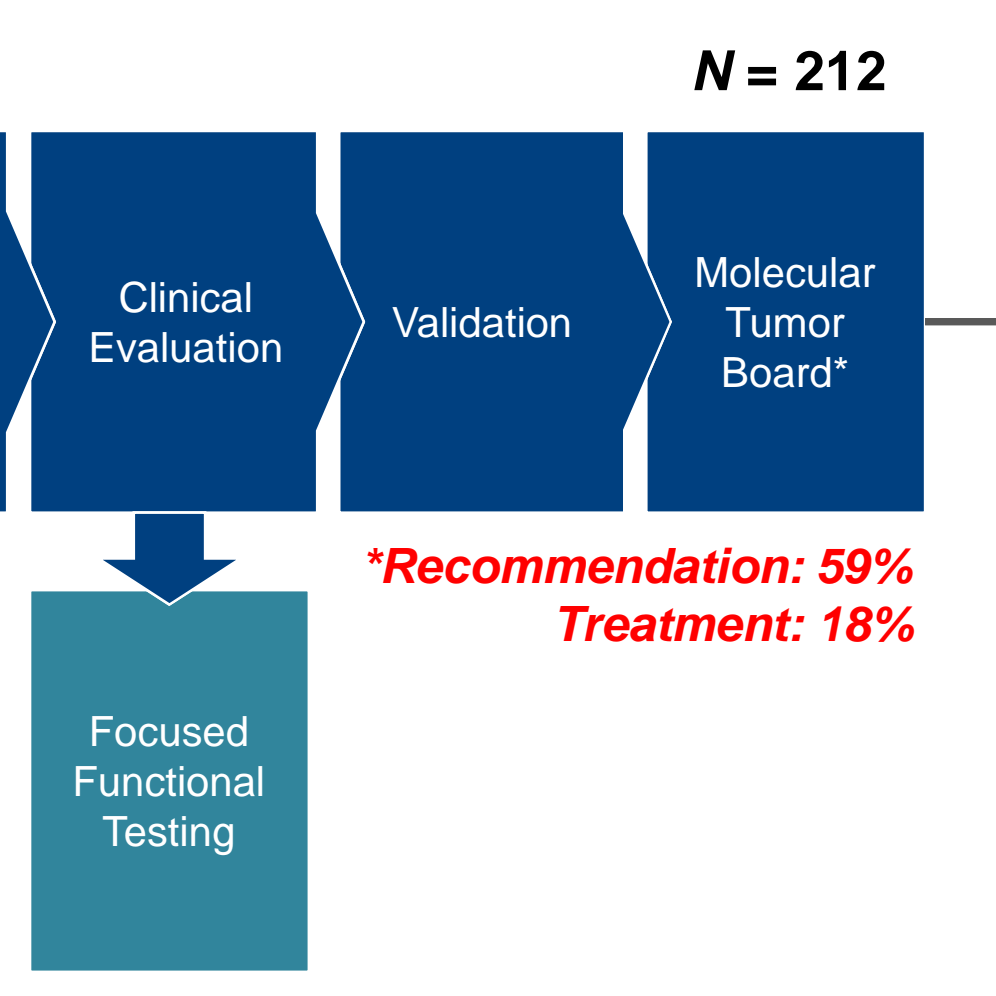

Treatment: $18 \%$

Focused

Functional

Testing

\section{Potential Strategies for Interventional Clinical Trial(s)}

- Fill baskets and collect information on treatment outcome

- Identify successful baskets (OR or SD for $\geq 6$ months in $\geq 2 / 10$ patients)

- Define trial design, for example:

- "Randomization" between patients receiving genomics-guided treatment and patients receiving standard of care (due to logistical or regulatory reasons etc.)

In collaboration with Annette Kopp-Schneider and Axel Benner, Division of Biostatistics, DKFZ 


\section{NCT / DKFZ}

Translational Oncology

Stefan Fröhling, Christoph Heining,

Stefan Gröschel, Hanno Glimm

Medical Oncology

Dirk Jäger and Team

\section{NCT POP / DKFZ-HIPO}

Sample Processing / Coordination

Christina Geörg, Katja Oehme, Daniela Richter, Katja Beck

Board of Directors

Peter Lichter, Roland Eils,

Christof von Kalle

\section{DKFZ}

Sequencing Core Facility Stephan Wolf and Team

Clinical Bioinformatics

Barbara Hutter, Benedikt Brors

Heidelberg University

Molecular Pathology

Volker Endris, Roland Penzel, Albrecht Stenzinger, Wilko

Weichert, Peter Schirmacher

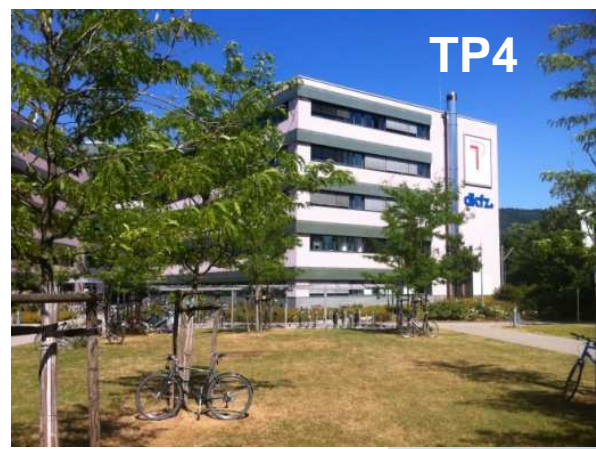

TP3

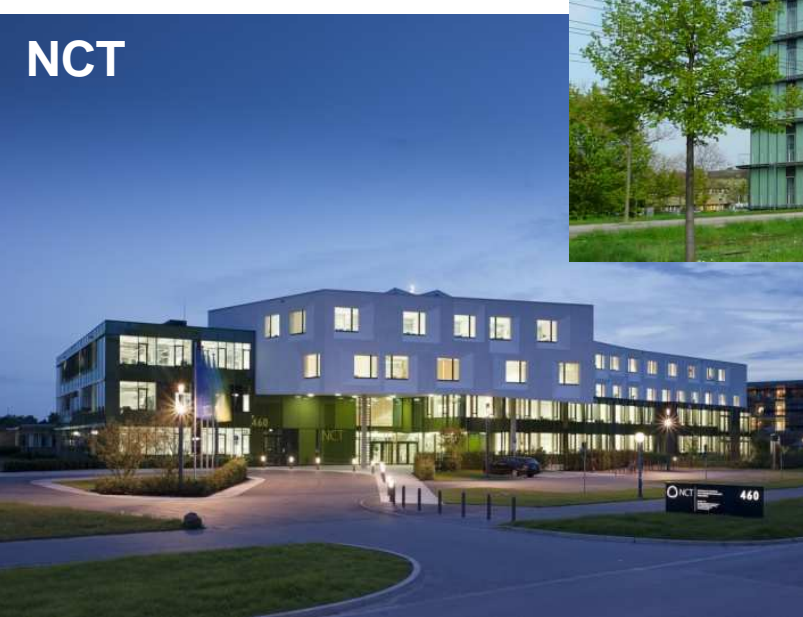

DKFZ-HIPO

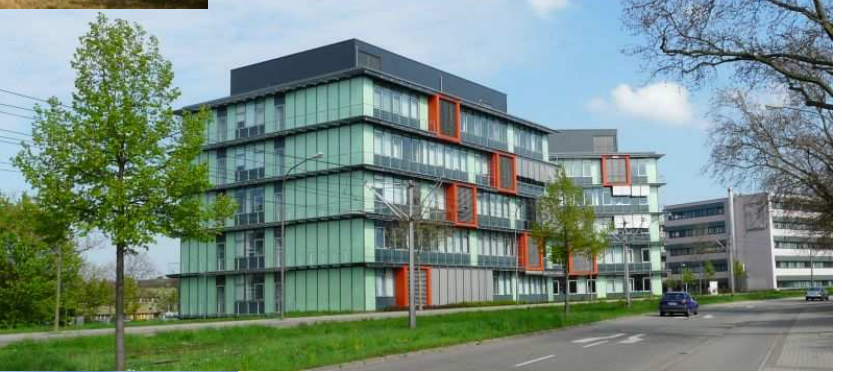

Pathology
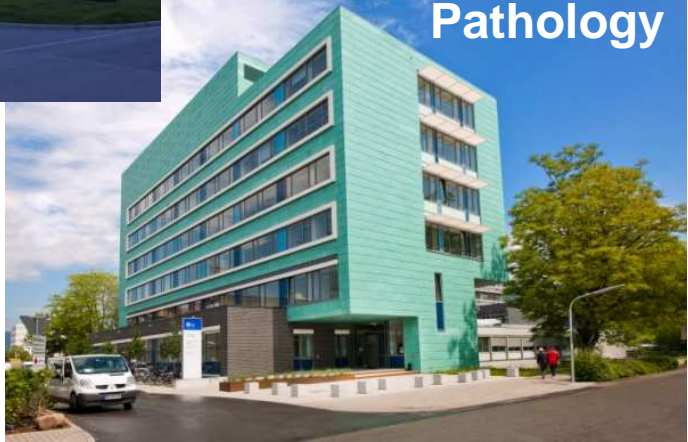